\title{
SECTOR CROSS-REFERENCE
}

This appendix provides a cross-referenced display of how sectors funded by the Iraq Relief and Reconstruction Funds (IRRF 1 and IRRF 2), the Iraq Security Forces Fund (ISFF), the Economic Support Fund (ESF), and the Commander's Emergency Response Fund (CERP) relate to the SIGIRdefined sectors. 
TABLE D.1

SECtor CRoss-Reference

\begin{tabular}{|c|c|c|c|c|c|c|}
\hline AREA & SIGIR SECTOR & ESF PROGRAMS & IRRF 2 SECTORS & CERP PROJECT TYPES & ISFF & IRRF 1 PROG RAMS \\
\hline Security & Security and Justice & $\begin{array}{l}\text { Marla Ruzicka Iraq War } \\
\text { Victims Fund (transferred to } \\
\text { IRRF) } \\
\text { Regime Crimes Liaison } \\
\text { Office }\end{array}$ & $\begin{array}{l}\text { Security and Law } \\
\text { Enforcement } \\
\text { Justice, Public Safety, } \\
\text { and Civil Society }\end{array}$ & $\begin{array}{l}\text { Battle Damage } \\
\text { Civic Infrastructure Repair } \\
\text { Protective Measures } \\
\text { Detainee Release } \\
\text { Payments } \\
\text { Law \& Governance } \\
\text { Condolence Payments } \\
\text { Civic Cleanup Activities }\end{array}$ & $\begin{array}{l}\text { All } \\
\text { Funding }\end{array}$ & $\begin{array}{l}\text { First Response Network/DIILS } \\
\text { Police/Prison Programs } \\
\text { Law Enforcement }\end{array}$ \\
\hline Economy & $\begin{array}{l}\text { Economic } \\
\text { Development }\end{array}$ & $\begin{array}{l}\text { Inma Private Sector } \\
\text { Agribusiness Development } \\
\text { Provincial Economic Growth } \\
\text { (PEG) Program } \\
\text { Targeted Development } \\
\text { Program } \\
\text { Izdihar } \\
\text { Economic Governance II, } \\
\text { Policy \& Regulatory Reforms } \\
\text { Infrastructure Security } \\
\text { Protection (ISP) - Private } \\
\text { Sector Development }\end{array}$ & $\begin{array}{l}\text { Private Sector } \\
\text { Development }\end{array}$ & $\begin{array}{l}\text { Food Production \& } \\
\text { Distribution } \\
\text { Agriculture } \\
\text { Economic, Financial, } \\
\text { and Management } \\
\text { Improvements } \\
\text { Civic Support Vehicles }\end{array}$ & $\mathrm{N} / \mathrm{A}$ & $\begin{array}{l}\text { Expand Economic } \\
\text { Opportunity } \\
\text { Technical Assistance } \\
\text { Technical Assistance/Training }\end{array}$ \\
\hline \multirow[t]{7}{*}{ Infrastructure } & Electricity & $\begin{array}{l}\text { O \& M Sustainment - } \\
\text { Electricity } \\
\text { Plant-Level Capacity } \\
\text { Development \& Technical } \\
\text { Training - Electrical } \\
\text { Infrastructure Security } \\
\text { Protection (ISP) - Electrical }\end{array}$ & Electricity Sector & Electricity & N/A & Restore Iraq Electricity (RIE) \\
\hline & Oil and Gas & $\begin{array}{l}\text { O \& M Sustainment - Oil } \\
\text { Infrastructure Security } \\
\text { Protection (ISP) - Oil }\end{array}$ & Oil Infrastructure & N/A & $\mathrm{N} / \mathrm{A}$ & Restore Iraq Oil (RIO) \\
\hline & Water & $\begin{array}{l}\text { O \& M Sustainment - PW \& } \\
\text { Water } \\
\text { Plant-Level Capacity } \\
\text { Development \& Technical } \\
\text { Training - PW \& Water } \\
\text { Infrastructure Security } \\
\text { Protection (ISP) - PW \& Water }\end{array}$ & $\begin{array}{l}\text { Water Resources and } \\
\text { Sanitation }\end{array}$ & Water \& Sanitation & N/A & N/A \\
\hline & Health Care & $\begin{array}{l}\text { O \& M Sustainment - Health } \\
\text { Plant-Level Capacity } \\
\text { Development \& Technical } \\
\text { Training - Health }\end{array}$ & Health Care & Health Care & N/A & N/A \\
\hline & $\begin{array}{l}\text { Transportation and } \\
\text { Communications }\end{array}$ & $\begin{array}{l}\text { O \& M Sustainment } \\
\text { - Transportation and } \\
\text { Communications } \\
\text { Plant-Level Capacity } \\
\text { Development \& Technical } \\
\text { Training - Communication } \\
\text { Plant-Level Capacity } \\
\text { Development \& Technical } \\
\text { Training - Transportation }\end{array}$ & $\begin{array}{l}\text { Transportation and } \\
\text { Communications } \\
\text { Roads, Bridges, and } \\
\text { Construction }\end{array}$ & $\begin{array}{l}\text { Transportation and } \\
\text { Telecommunications }\end{array}$ & N/A & N/A \\
\hline & Buildings & $\begin{array}{l}\text { O \& M Sustainment - } \\
\text { Buildings } \\
\text { Infrastructure Security } \\
\text { Protection (ISP) - Buildings }\end{array}$ & N/A & N/A & $\mathrm{N} / \mathrm{A}$ & $\mathrm{N} / \mathrm{A}$ \\
\hline & $\begin{array}{l}\text { General } \\
\text { Infrastructure }\end{array}$ & N/A & N/A & N/A & N/A & $\begin{array}{l}\text { Restore Economically Critical } \\
\text { Infrastructure }\end{array}$ \\
\hline
\end{tabular}




\section{SECTOR CROSS-REFERENCE}

AREA

SIGIR SECTOR

ESF PROGRAMS

IRRF 2 SECTORS

CERP PROJECT TYPES

ISFF

Governance

Capacity

National Capacity

Admin Expense (USAID, Other Humanitarian and N/A

Reconstruction Projects

IRRF 1 PROGRAMS

Development State)

Improve Efficiency and

Ministerial Capacity

Development

Accountability of Government

Community Stabilization

Program Support and

Program

Development of Gulf Region

PRT/PRDC

Administrative Expenses

PRT/QRF

\begin{tabular}{|c|c|c|c|c|c|}
\hline Democracy & $\begin{array}{l}\text { Community Action Program } \\
\text { Local Governance Program } \\
\text { Democracy and Civil Society }\end{array}$ & N/A & $\mathrm{N} / \mathrm{A}$ & N/A & $\mathrm{N} / \mathrm{A}$ \\
\hline \multirow{4}{*}{$\begin{array}{l}\text { Refugees, Human } \\
\text { Rights, and } \\
\text { Education }\end{array}$} & \multirow[t]{4}{*}{ Iraqi Refugees (Jordan) } & \multirow[t]{4}{*}{$\begin{array}{l}\text { Education, Refugees, } \\
\text { Human Rights }\end{array}$} & \multirow[t]{4}{*}{ Education } & \multirow[t]{4}{*}{$\mathrm{N} / \mathrm{A}$} & $\begin{array}{l}\text { Support Education, Health, } \\
\text { and Social Services }\end{array}$ \\
\hline & & & & & $\begin{array}{l}\text { Relief: Office of Foreign } \\
\text { Disaster Assistance }\end{array}$ \\
\hline & & & & & Relief Efforts \\
\hline & & & & & Humanitarian Demining \\
\hline
\end{tabular}

*** ESF Sector-level data for the O\&M Sustainment, Infrastructure Security Protection, and Plant-Level Capacity Development \& Technical Training programs, is available through the IRMS, ESF Cost to Complete Report, and will not reconcile with the total program figures received from GRD. 